\title{
APPLICATIONS OF SIMULATION IN PROGRESS REPORTING AND CONTROL
}

\author{
Nader N. Chehayeb \\ Simaan M. AbouRizk \\ Department of Civil Engineering \\ University of Alberta \\ Edmonton, Alberta, T6G 2G7, CANADA
}

\begin{abstract}
This paper describes the development of SimCon (SIMulation-based project CONtrol) system that provides the basis for implementing progress reporting and control in a simulation model. The issues considered in SimCon include developing a reporting by exception capability from a hierarchical breakdown structure that will minimize progress reporting at the very detailed level. Simulation constructs are developed to store project progress information and simplify the logical linking of simulation processes. SimCon is implemented using object oriented concepts, event driven programming, relational databases, and a simulation engine. The objective of this research is improving construction management productivity in progress reporting and control and improving decision making by incorporating detailed resource level interactions in project modeling.
\end{abstract}

\section{INTRODUCTION AND BACKGROUND}

This paper presents a methodology for implementing progress reporting and project control using a simulation model. Most construction project planning methods employ some form of cost and schedule control. Progress reporting involves the recording of construction achievements for detection of deviations from actual plan and for forecasting project performance. Any construction project can be comprised of summary level activities for higher level management (long term planning) and/or very detailed level of activities and sub-activities with numerous cost accounts for day to day control (short term planning). Representing project information and level of detail in activities depends on control objectives, available collection tools, and justifiable reasoning for the recommended level of detail. Short term planning requires numerous detailed level activities showing interaction of resources, required quantities, expected production rates, and personnel involved. On the other hand incorporating such a highly detailed level of planning in a schedule places an overburden on the time and cost required to update the plans during project execution.

A number of systems currently exist for representing project information. Some of the current management systems provide a mechanism to summarize information at various levels of detail using techniques such as a work breakdown structure (WBS). A WBS is an organization of project activities in a hierarchical format. Development of activities in a WBS helps to organize the structure of activities for project summary evaluation and reporting for upper level management.

The use of system simulation to represent activities at the lowest level in a WBS provides construction managers with advanced project control mechanisms for better management of resources and risks. There has been a number of attempts to incorporate simulation at the project level for project planning. An organized breakdown structure of a project is composed of operations, processes, and individual tasks as suggested in Halpin and Woodhead (1976) (i.e., a project is made up of individual operations consisting of individual processes which are made up of individual tasks). Dabbas (1981) describes a project planning technique that integrates process level simulation with Critical Path Method (CPM) network scheduling. Dabbas attempts to improve decision making of higher level management by incorporating dynamic interactions of resources at the site level. However, resources between separate processes were not integrated.

Riggs (1989) in his literature review provides a summary of the attempts made to use simulation modeling for project planning. An object oriented 
discrete event simulation network, CIPROS, was developed by Odeh et al. (1992). CIPROS does not provide process-level interactions in its hierarchical modeling tool for planning projects. Interaction of processes in a hierarchical simulation-based project planning (HSM) was achieved by Sawhney (1994). A project plan can be developed in HSM using a symbolic graphical format. HSM is limited to planning a project and does not provide progress reporting and feedback nor provide a methodology to represent activity relationships other than time.

The use of simulation improves decision-making in problems related to construction industry and provides a better mechanism for advanced project management. However, the effort of reporting progress at the process level (lowest level in a WBS) in a construction project can not be justified. Tremendous input of data is required at the process level in a simulation model for progress control. In general, more tasks and resources have to be updated (report on progress during construction) in a simulation model than in a CPM model.

Controlling all details in a project is required only if a project is not progressing according to plan (control in this paper is used to mean progress reporting and feedback within a scheduling system). "A sure sign of schedule planning failure occurs when project control engineers can be found spending all their time updating schedules ..." as stated by the Construction Industry Institute (1987). The author's experience with a major general contractor in Canada, PCL Constructors Inc., shows that there is not enough time to collect data to support current scheduling techniques

\section{PROJECT MODELING IN SIMCON}

The basic fundamentals of current progress reporting mechanisms focus on reporting progress at the lowest level of detail in a WBS. The methodology of development of a WBS for a construction project greatly affects the progress reporting mechanisms. This paper presents a new methodology of hierarchical representation of project information that allows simulation-based project control of a project. The objective is to develop a SIMulation-based project CONtrol (SimCon) system that improves construction management productivity in progress reporting and project control. SimCon is implemented using object oriented concepts and event driven programming. The object oriented concepts were utilized in the graphical user interface of constructing the simulation processes. Graphical objects were created in the Visual $\mathrm{C}++{ }^{\mathrm{TM}}$ environment and then exported into Visual Basic ${ }^{\mathrm{TM}}$ (event driven programming). Relational database was implemented using ACCESS ${ }^{\mathrm{TM}}$ engine to store project related information. The simulation system that is currently being used to test and validate the concepts of SimCon is SLAM II (Pristker 1989). The main goals of SimCon include the following:

- Provide a hierarchical breakdown structure that will minimize the effort of reporting progress at the very detailed level through reporting by exception.

- Provide simulation constructs to store project progress information and simplify the logical linking of simulation processes.

- Automate the suggested methodology in a form that allows validation and testing of the concepts using an actual case study.

The implementation of SimCon addresses five important issues: 1) cost center identification; 2) location breakdown structure; 3) implemented construction methods and project schedule; 4) progress reporting mechanism to track by work responsibility; and 5) construction processes' logical sequencing. Figure 1 shows a general schematic overview of SimCon's hierarchic system. There are a number of advantages for using this hierarchy. The basic advantages are the following: 1) providing a short term planning tool from the very detailed representation at the process level; 2) providing a production-based identification structure and a better way to link processes, classify problem areas, and track project progress; and 3) providing progress reporting by exception capability to update a project control schedule at a higher level in a production breakdown structure.

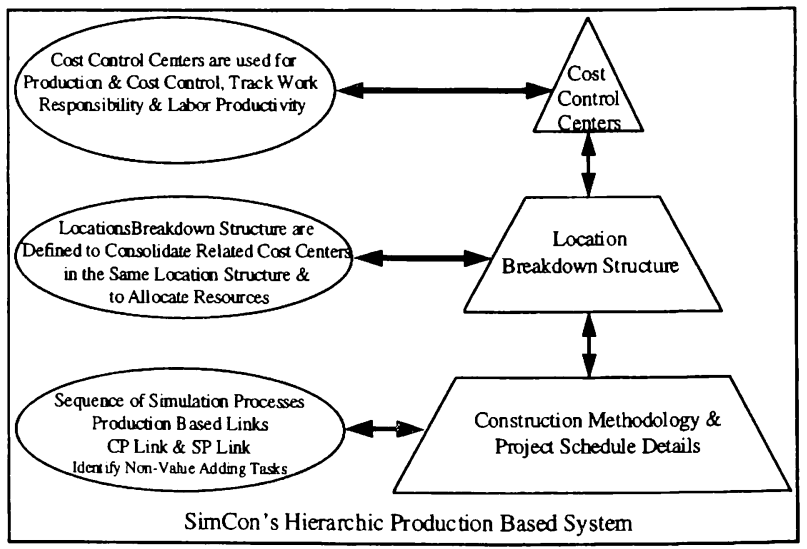

Figure 1: A Schematic Overview of SimCon's Hierarchic System. 


\subsection{Cost and Crew Center Identification}

Cost control centers are identified by the user and depend on the type of project and general contract requirements. For building construction, cost control centers can build on the standard master list of cost accounts as shown in Figure 2. This format is used by many construction companies to track cost expenditures and labor productivity in building projects. A modeling scheme that builds on this format will help to bridge the gap between current cost control techniques and new modeling techniques. A hierarchical cost code structure is used for estimating, planning, progress reporting and feedback, and for maintaining historical records for future projects. However, cost centers do not show specific locations for detailed control purposes. A further drawback of such a breakdown is the inability to link and sequence activities in a logical flow diagram.

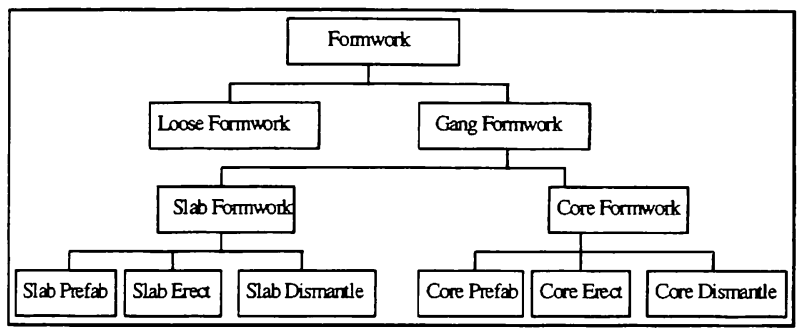

Figure 2: A Sample of Cost Control Centers for Building Construction

\subsection{Location Breakdown Structure}

Location breakdown structure is used to link construction methods and project schedule to associated cost centers. To simplify defining locations a hierarchical location breakdown structure (LBS) was used as shown in Figure 3. Specific location breakdown structures in the lower level of the location hierarchy inherit their parent's characteristics. An LBS is used to allocate resources and to define specific attributes such as space constraints and location access requirements. Resources naturally occupy space in a specific location. For example, a project consisting of two buildings can be allocated two cranes; one for each building. On the project level a truck can be shared by both buildings, hence, allocated at a higher level in the hierarchical location breakdown structure. Furthermore, an LBS is used as a mechanism to group together cost centers with the same location. Similarly an LBS is used to group construction methods in the same location. The location structure as shown in Figure 3 can be used to report on schedule progress in a project by location.

Russell and Wong (1993) suggest that the concept of work locations be thought of in general terms, embracing off-site processes and as well as on-site physical locations. Off-site processes which are usually contained in the same schedule include different stages of design, permits, and procurement. Figure 3 shows an example of on-site physical locations. Russell and Wong further suggest that a hierarchy of locations would be ideal for large scale facilities and/or microplanning (this feature was not implemented in their research work).

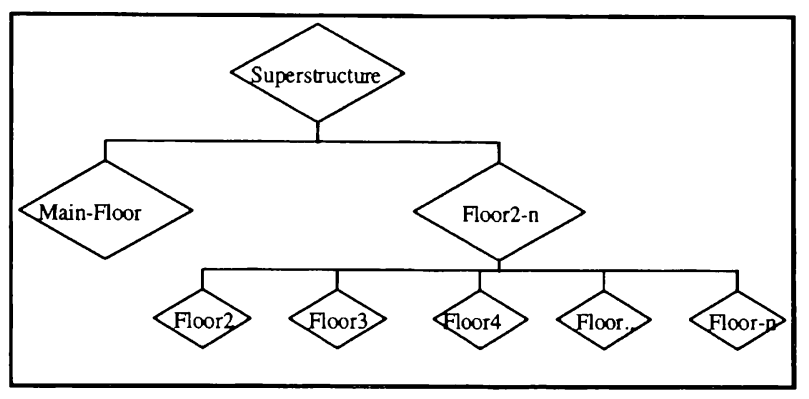

Figure 3: A Schematic View of Location Breakdown Structure Concept for On-Site Processes

\subsection{Construction Processes and Methods}

Construction methods are constructed and identified in the sequencing of tasks in a simulation process. SimCon builds on the standard simulation elements of CYCLONE which was developed by Halpin (1973, 1990). Some additional elements besides the CYCLONE elements were required to implement the SimCon concept. The additional elements are basically used to keep track of progress achieved and to simplify the linking of processes.

Processes can be either retrieved from SimCon's library database of processes or constructed by the user using SimCon's graphical interface in the computer prototype system. Construction processes are required to be attached to an LBS because construction has to be done in a specific location. If a process is attached to a parent LBS then repetitive or cyclic sequencing of tasks can be used for completing the processes at "children" locations. Dynamic variables included in a simulation process include: number and type of resources, task durations, and production required. These variables are assigned from outside individual processes through the use of attributes on specific activities. In this regard, 
simulation processes will mainly be constructed by assigning actual values to the variables of each activity's duration, resource usage, and production. These variables are controlled by passing values through special subroutine functions for every process.

In any type of construction, activities can be separated into either a repetitive process (rp-process) or a non repetitive process (np-process). An rp-process includes any cyclic type process such as excavation, slab reshoring, slab rebar placing, slab concrete pouring, and so on. An np-process includes any non cyclic activity such as obtaining permits, installing electrical generator, placing a sign, approving shop drawings, and so on. An rp-process is controlled on production basis where as an np-process is controlled on time basis.

Logical flow diagram in a process and other project environment constraints are considered to be static variables. However, a user can change a process logic through a "drag and drop" mechanism in the computer graphical interface. A typical simulation process showing interaction of resources and construction sequence logic at the process level is shown in Figure 4. Processes defined in SimCon's process library can be used at different locations and do not store actual values in any of the activities or resources.

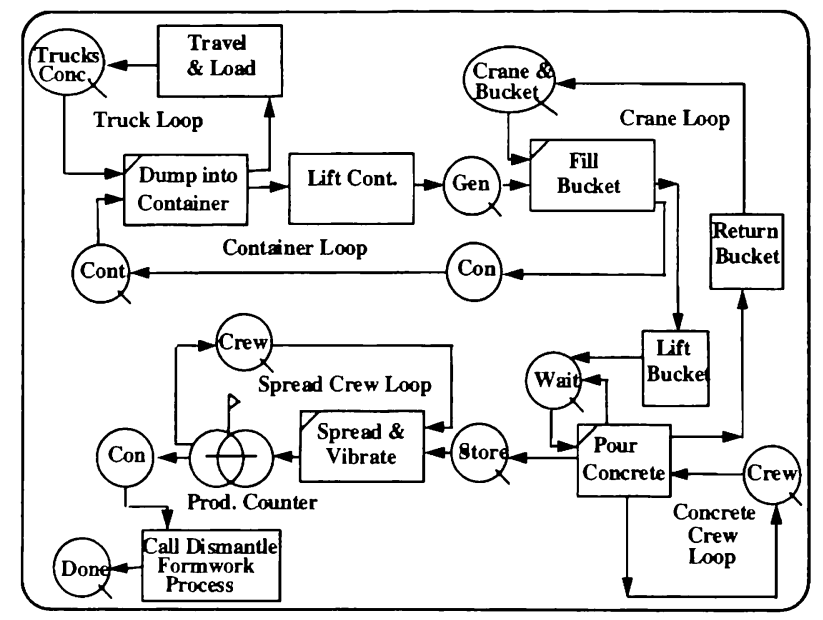

Figure 4: A Schematic View of a Within-Process Production Counter in a Simulation Process

A production counter is used to control production within and between processes. A typical within-process production counter (wp-counter) as shown in Figure 4 keeps track of production achieved and converts actual production to a simulation counter. A typical betweenprocess production counter (bp-counter) is shown in Figure 5. It keeps track of internal processes by re- initializing specified variables after every cycle. A bpcounter can also be used to track locations separately.

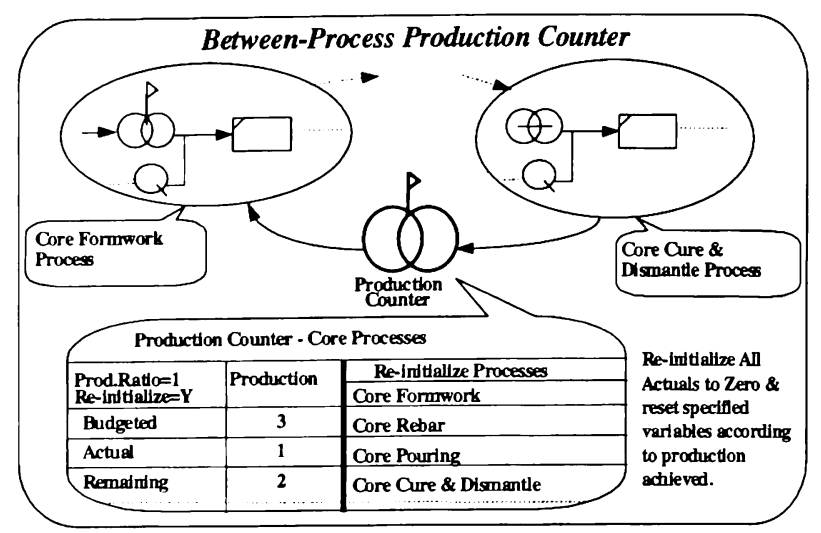

Figure 5: A Schematic View of a Between-Process Production Counter in a Simulation Process

\subsection{Logic Flow Diagram Between Processes}

Current activity-based systems provide time as the only constraint between activities. This paper addresses the issue of providing activity relationships based on production and incorporating these relationships in a progress reporting system. Many researchers have identified the need for better mechanisms to linking activities. Pultar (1990) realized as many other researchers (Hendrickson et al. 1987; and Fiedler 1985) that the lead/lag factors in terms of time are actually meant to be in terms of progress. Pultar attempted to provide a solution by using progress charts and Gantt (bar) charts. Although new relationships were suggested by using non-linear relationships between activities, Pultar was still using time-based relationships. Relationships between activities were still based on time and do not consider other means for progress relationships.

In SimCon two types of links are used: 1) SP-Link (single production link); and 2) CP-Link (continuous production link). A SP-Link is a simple one-time link between processes. A SP-Link is used to determine the production required before the next process can start. If the next process starts at completion of a predecessor process then SP-Link is equal to total actual production at the predecessor process. For example, placing steel process will start when a certain production is achieved in its predecessor formwork erection process.

A CP-Link is a more complex type relationship requiring multiple and continuous links between two processes. A CP-Link is used to sequence cyclic 
processes continuously by specifying a buffer zone between two processes. The buffer zone is used to control both the predecessor process and the successor process to make sure that the spacing in terms of production between both processes is in the range specified in the CP-Link. Linking of simulation processes can become very complex by certain logical constraints. For example the repetitive processes of a paving operation should not be represented as shown in Figure $6 \mathrm{a}$ if the excavator, in such a scenario, is required to be working on the next section even if compaction of the current section is not completed. Representing such sequencing as shown in Figure $6 \mathrm{~b}$ requires an experienced simulation analyst and considerable time to model a project to make sure that placing subbase is not done before excavating and leveling. As demonstrated in Figure $6 \mathrm{~b}$ placing subbase process is continuously following excavate and level process. The CP-Link makes sure that subbase process starts after a specified production in excavation and that it does not exceed a certain limit to maintain the sequence of production in both processes.

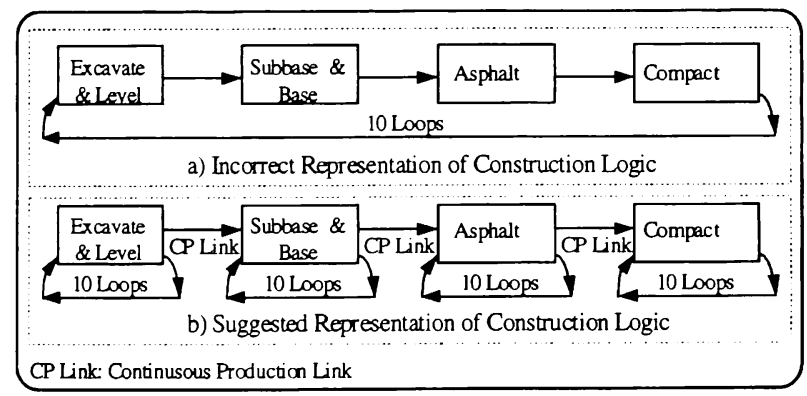

Figure 6: Schematic View for Modeling a Paving Operation; a) Incorrect Way to Represent Cyclic Operations; b) Suggested Representation of Construction logic.

\section{PROGRESS REPORTING MECHANISMS IN SIMCON}

Information about cost performance and project progress is reported on field reports and tabulated in desired project and labor cost reports. Such reports are used to measure actual achievement and to forecast project performance through the comparison with budgeted plans and other historical records. If on comparison the actual numbers do not conform with the budgeted numbers then the source of the problem must be identified and a corrective action suggested. The source of problems, such as cost overrun or schedule delay, involves determining responsible personnel (internal) or environmental causes (external). Following that, a corrective action can be suggested by implementing different scenarios on the simulation model for best outcome.

Progress reporting mechanisms in SimCon include production/resource-based reporting from a location breakdown structure. Exceptional progress reporting mechanisms in SimCon imply reporting progress at a higher level in the project's hierarchical breakdown structure. Exceptional progress reporting is necessary and especially in simulation modeling where numerous interaction of resources are to be considered. Moreover, durations of activities in many cases are much less than the updating frequency and especially for repetitive processes.

\subsection{Production-Based Reporting}

Production at the process level can be generated from a cyclic activity or a non cyclic activity. Production represents a specific output that is modeled in repetitive processes (rp-process) by the continuous cycling of specific tasks. In a rp-process production achieved to date will be transformed automatically using SimCon's production counter into a usable simulation parameter to update progress achieved.

In non repetitive processes (np-process) time represents output that is measured from a specific task. The earned value concept suggested by Moselhi (1993) is used in SimCon to report progress on a np-process as shown in Table 1 . The generalized progress measurement system is shown in Table 1. For example, for short duration and low cost activity, it is suggested that a zero - one hundred percent rule be used. That is the activity is zero percent complete until 'hand over' (i.e., activity becomes one hundred percent complete).

Table 1: Generalized Progress Measurement System (Moselhi 1993)

\begin{tabular}{|c||c|c|c|}
\hline \multicolumn{1}{|c||}{ Cost } & \multicolumn{3}{c|}{ Duration } \\
\cline { 2 - 4 } & Very Short & Short & Long \\
\hline \hline Low & $0-100$ & $50-100$ & $20-100$ \\
\hline \multirow{2}{*}{ High } & $0-100$ & $20-100$ & $20-100$ \\
& & $30-100$ & \\
\hline
\end{tabular}

Production in a np-process is considered to be equal to one. Production is measured differently for various processes in both rp-process and np-process. Reporting on production achieved in a specific process is used to update a schedule in SimCon. 


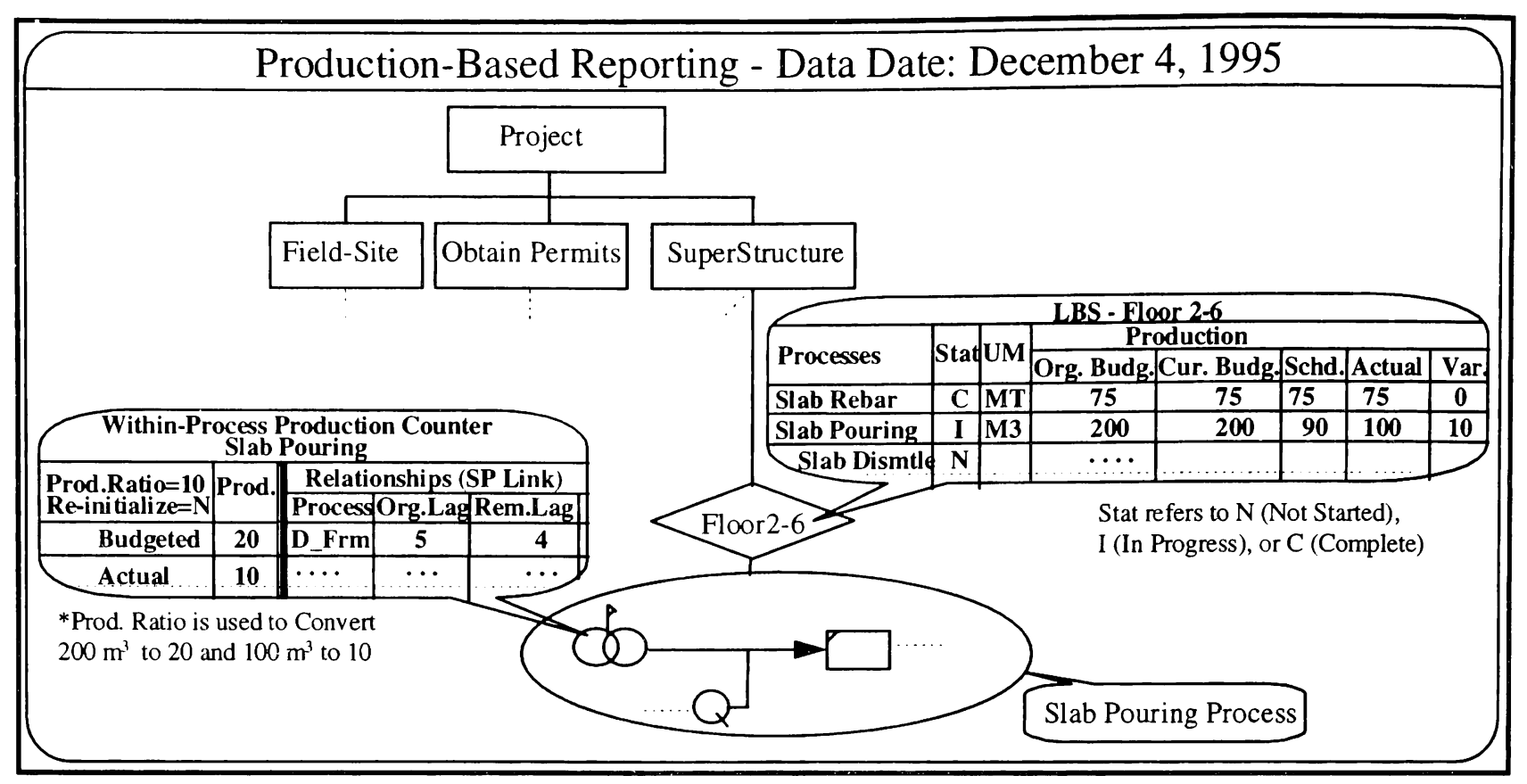

Figure 7: Schematic View of Production-Based Reporting in SimCon

Dynamic and static variables that are initially defined to construct a project plan are stored as budgeted estimates in SimCon's database. Budgeted dynamic variables are estimated at the process level. Progress reporting on a specific location depends on the type of estimate available at the process level. Production on cyclic activities is updated by changing dynamic variables stored in a production counter. A wp-counter, as shown in Figure 7, is used to store budgeted production, actual production completed to date, and a ratio that converts actual reported production from field to a standard simulation number. For example, $200 \mathrm{~m}^{3}$ of concrete can be represented by a production counter to be 20 loops (200/10). Similarly, actual production reported in the field of $100 \mathrm{~m}^{3}$ is converted into a simulation counter of $10(100 / 10)$. The production ratio used in the wp-counter is set equal to 10.

\subsection{Resource-Based Reporting}

Recording and tracking progress in a project is directly affected by the way information is presented to project controllers as described by Sanvido and Paulson (1992) (a controller can be a foreman, superintendent, or project manager). Sanvido and Paulson developed a conceptual construction process model that improves feedback systems, resource supply systems, and construction method and planning functions. Hierarchy operation in the field, such as superintendent above foreman and foreman above craftsman, provide a project control system. In this process a controller is in charge of the resource that he uses himself. If a resource is shared by multiple areas and processes then a higher level controller is required to be in control of the resource. For example, if tower crane is allocated to multiple locations and disciplines then a superintendent will control it. A controller must plan and coordinate the work sequence and resources at one level below.

SimCon addresses the reporting of progress on resource utilization in a similar methodology as described by Sanvido and Paulson (1992). Reporting on resource utilization is implemented by exception. This means that a specific resource performance can be evaluated for the entire project. If performance is not satisfactory then a detailed analysis can be performed at the process level. Statistical reports from simulation include percentage idle time and busy time for each resource. Resources can be either of $\mathrm{C}$ (contractor crew composition), $\mathrm{E}$ (equipment), or $\mathrm{S}$ (subtrade crew composition). A schematic view of resource evaluation in SimCon is shown in Figure 8. 


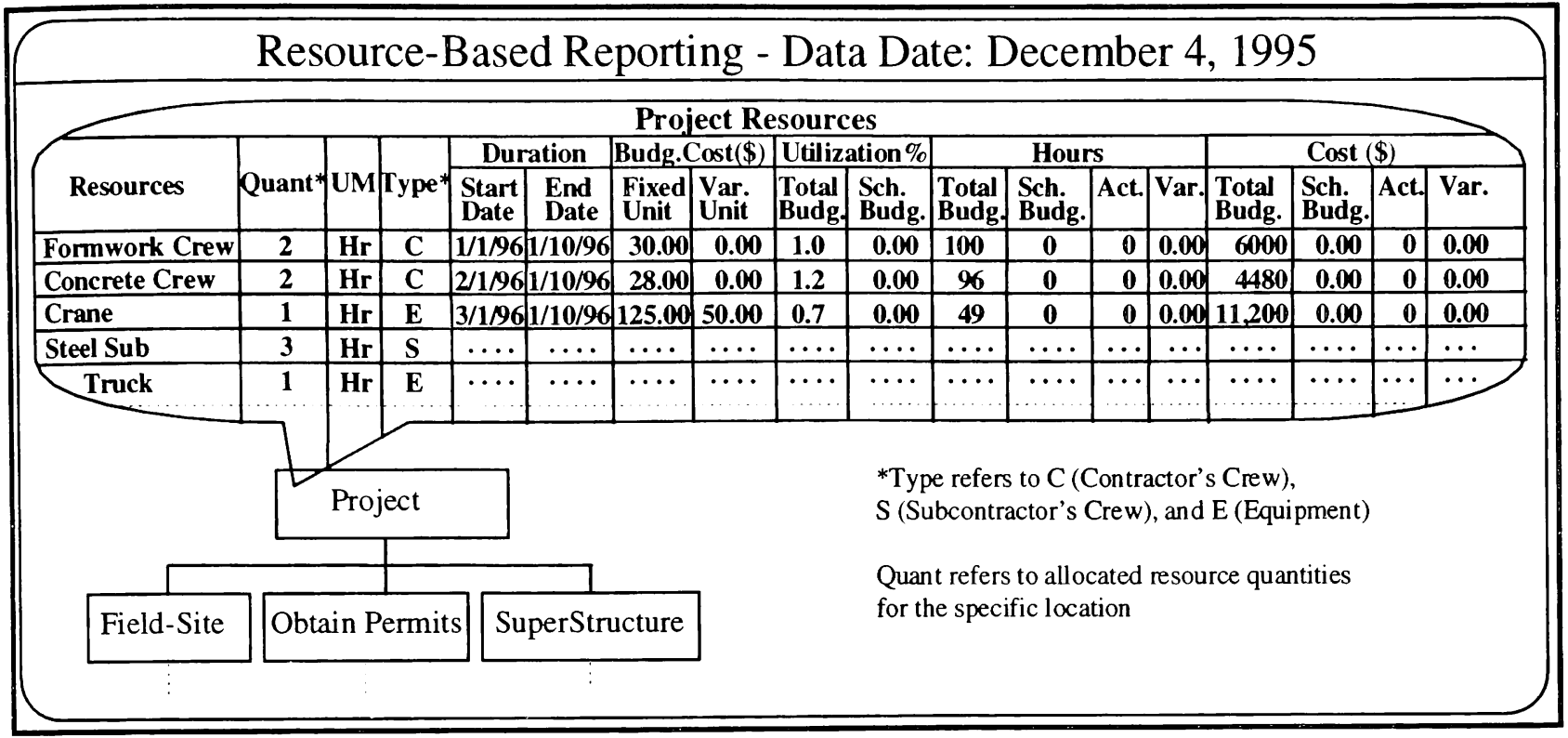

Figure 8: Schematic View of Resource-Based Progress Reporting in SimCon

The equations used in the resource-based report are as follows. Total budgeted utilization (TBU) and scheduled budgeted utilization (SBU) are internally calculated by the simulation engine. SBU is equal to the planned utilization of a resource up to the data date. Total budgeted hours is equal to TBU times resource duration. Resource duration is equal to end date minus start date of a specific resource. Total budgeted cost of a resource is equal to fixed unit cost multiplied by resource duration plus variable unit cost multiplied by total budgeted hours.

\section{FINDINGS AND CONCLUSIONS}

The focus of this paper was on applying advanced project control techniques and minimizing the time spent on progress reporting at the very detailed level of a hierarchical breakdown structure. There are a number of advantages for using simulation for project control. Simulation modeling improves the decision-making of upper level construction management by providing a mechanism to evaluate non-value added tasks and resource utilization. Traditional scheduling control tools lack the required interactions with the lower level management where the dynamic nature of a construction process and its complex interrelated components interact; basically, the level at which resources interact.
The problem with applying simulation modeling is the tremendous data support required for progress reporting. This research work attempts to overcome this handicap by integrating project components in a hierarchical production breakdown structure to facilitate exceptional progress reporting at higher levels. This involves reporting on production achieved and resources consumed at a higher level in the hierarchy.

Advanced project control greatly assists contractors in better control of resources, better management of production, and exceptional progress reporting capabilities. A prototype computer system currently exists that implements the above concepts but was not included due to space limitations.

\section{ACKNOWLEDGMENTS}

We thank Mr. Ian Kermack for many enlightening discussions related to progress reporting and project control. Thanks are also due to Professors Peter Dozzi and William Weir for their recommendations and support. This work was supported in part by the Natural Science and Engineering Research Council (NSERC) grant of Canada under Grant number CRD166483.

\section{REFERENCES}

Construction Industry Institute. 1987. Project Control for Construction. Publication 5-6, Bureau of 
Engineering Research. The University of Texas at Austin.

Dabbas M. 1981. Computerized Decision Making in Construction. Ph.D. Dissertation, Georgia Institute of Technology, Atlanta, Georgia.

Fiedler, K. 1985. Special Conditions for Time Scheduling of Building Modernization Projects. Proceedings of the Eighth Conference on INTERNET. 2: 461 - 468. Dutch Project Management Society

Halpin, D. W. 1973. An Investigation of the Use of Simulation Networks for Modeling Construction Operations. Ph.D. Dissertation, University of Illinois, at Urbana-Champaign, Illinois.

Halpin D and R. Woodhead. 1976. Planning and Analysis of construction Operations. New York, NY: John Wiley and Sons.

Halpin D W. 1990. Micro CYCLONE System Manual. Division of Construction Engineering and Management, Purdue University, West Lafayette, IN.

Hendrickson, C., D. Martinelli, and D. Rehak. 1987. Hierarchical Rule-Based Activity Duration Estimation. ASCE Journal of the Construction Division, 113: 288 - 301.

Odeh A.M., I. D. Tommelein, and R. I. Carr. 1992. Knowledge-Based Simulation of Construction Plans. Proceedings of the Eighth ASCE Conference on Computing in Civil Engineering, Dallas, Texas, 1042-1049.

Moselhi, O. 1993. Applied Earned Value for Project Control. CIB W-65, Trinidad. 869 - 879.

Pritsker A.A.B., C. E. Sigal, and R.D.J. Hammesfahr. 1989. SLAM II - Network Models for Decision Support. New Jersey: Prentice-Hall Inc.

Pultar, M. 1990. Progress-Based Construction Scheduling. ASCE Journal of the Construction Division, 116: 670 - 688.

Riggs, L.S. 1989. Risk Management in CPM Network. MicroComputers in Civil Engineering. 3: 229-235. Elsevier Applied Science

Russell, Alan D. and W. C. M. Wong. 1993. A New Generation of Planning Structures. ASCE Journal of Construction Engineering and Management, 119: $196-214$.

Sanvido, V. E., and B. Paulson. 1992. Site-Level Construction Information System. ASCE Journal of Construction Engineering and Management, 118: $701-715$.

Sawhney, A. 1994. Simulation-Based Planning for Construction. Ph.D. Dissertation, Department of Civil Engineering, University of Alberta, Edmonton.

\section{AUTHOR BIOGRAPHIES}

NADER N. CHEHAYEB is a Ph.D. candidate in the Department of Civil Engineering at the University of Alberta. He received a B.Eng. degree in Civil Engineering from the American University of Beirut in 1990 and received his M.Eng. in Civil Engineering from the University of Alberta in 1991. His research interests are focused on the application of computers for advanced project management, particularly the project control phase of a construction project.

SIMAAN M. ABOURIZK is an Associate Professor in the Department of Civil Engineering at the University of Alberta. He received a B.S. degree in Civil Engineering in 1984 from Georgia Institute of Technology and received his M.S. in 1985 in Civil Engineering from Georgia Institute of Technology. He received his Ph.D. degree in Civil Engineering from Purdue University in 1990. His research interests are focused on the application and modeling of simulation systems for construction project management. Dr. AbouRizk currently holds the Alberta Construction Industry Professorship and leads a research program in collaboration with a number of construction companies. 